
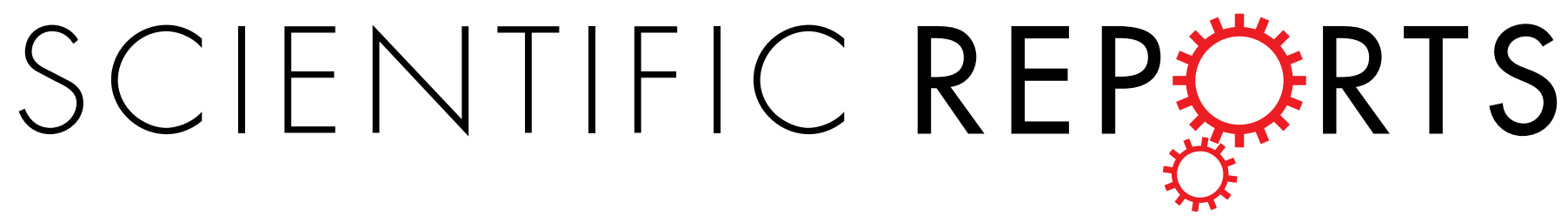

Corrected: Author Correction

Received: 11 October 2018

Accepted: 28 April 2019

Published online: 07 May 2019

\section{Thymoquinone therapy} remediates elevated brain tissue inflammatory mediators induced by chronic administration of food preservatives

\begin{abstract}
Ahmed Mohsen Hamdan $\mathbb{D}^{1}$, Mohammed M. Al-Gayyar ${ }^{2,3}$, Mohamed E. E. Shams ${ }^{4,5}$, Udai Salamh Alshaman ${ }^{1}$, Kousalya Prabahar ${ }^{1}$, Alaa Bagalagel ${ }^{1,6}$, Reem Diri ${ }^{6}$, Ahmad O. Noor \& Diena Almasri6

Continuous exposure to preservatives such as nitrite salts has deleterious effects on different organs. Meanwhile, Nigella sativa oil can remediate such organ dysfunction. Here, we studied the effect of consumption of thymoquinone (TQ); the main component of Nigella sativa oil on the brain damage induced by sodium nitrite. Forty adult male rats were daily given oral gavage of sodium nitrite $(80 \mathrm{mg} / \mathrm{kg})$ with or without thymoquinone $(50 \mathrm{mg} / \mathrm{kg})$. Oxidative stress, cytokines of inflammation, fibrotic elements and apoptotic markers in brain tissue were measured. Exposure to sodium nitrite (SN) resulted in increased levels of malondialdehyde, TGF- $\beta$, c-reactive protein, NF- $\kappa$ B, TNF- $\alpha$, IL-1 $\beta$ and caspase- 3 associated with reduced levels of glutathione, cytochrome c oxidase, Nrf2 and IL-10. However, exposure of rats' brain tissues to thymoquinone resulted ameliorated all these effects. In conclusion, thymoquinone remediates sodium nitrite-induced brain impairment through several mechanisms including attenuation of oxidative stress, retrieving the reduced concentration of glutathione, blocks elevated levels of pro-inflammatory cytokines, restores cytochrome c oxidase activity, and reducing the apoptosis markers in the brain tissues of rats.
\end{abstract}

Food additives are considered the main problem in issue of food safety. Individuals actually used food additives and suggested that additives should be exposed to toxicological evaluation and appropriate pharmacological testing. Routinely used food additives for fish and meat includes sodium nitrite. Sodium nitrite (SN) is a famous inorganic water-soluble salt, with $\mathrm{E}$ number E250. In a concentration above $80 \mu \mathrm{M}$, it inhibits the iron-sulfur clusters required for the energy metabolism essential for the growth of Clostridium botulinum in cooled meats ${ }^{1}$. Besides, it reacts with hemoglobin and metal ions leading to effectively delaying the oxidative rancidity. $\mathrm{SN}$ also chelates free radicals terminating the cycle of lipid oxidation ${ }^{2}$. Moreover, $\mathrm{SN}$, in a concentration of 2 to 14 parts per million, gives the meat its desirable red color by forming nitrosylating compounds that latterly interacts with myoglobin of the meat producing the red color ${ }^{3}$. However, for the sake of prolonging the duration of that red color, obvious greater concentrations of $\mathrm{SN}$ are needed ${ }^{2}$. It is not recommended to be used as a single component. European Union recommends its use as a $0.6 \%$ mixture.

$\mathrm{SN}$ is toxic for bacteria and animals, in high amounts, including humans. The LD50 of sodium nitrite for rats is $180 \mathrm{mg} / \mathrm{kg}$ and for human is $71 \mathrm{mg} / \mathrm{kg}$. An average person, consuming more than $4.615 \mathrm{~g}$ of SN per day, may result in death ${ }^{4}$. High-risk categories, as infants and children, consume high concentrations of nitrites has been stated

${ }^{1}$ Department of Pharmacy Practice, Faculty of Pharmacy, University of Tabuk, Tabuk, Saudi Arabia. ${ }^{2}$ Department of Pharmaceutical Chemistry, Faculty of Pharmacy, University of Tabuk, Tabuk, Saudi Arabia. ${ }^{3}$ Department of Biochemistry, Faculty of Pharmacy, Mansoura University, Mansoura, Egypt. ${ }^{4}$ Department of Pharmaceutics, Faculty of Pharmacy, Mansoura University, Mansoura, Egypt. ${ }^{5}$ Department of Pharmaceutics and Pharmacy Practice, Oman College of Health Sciences, Pharmacy Program, Ministry of Health, Muscat, Oman. ${ }^{6}$ Department of Pharmacy Practice, Faculty of Pharmacy, Kind Abdulaziz University, Jeddah, Saudi Arabia. Correspondence and requests for materials should be addressed to A.M.H. (email: a_hamdan@ut.edu.sa) 
B

TQ treated

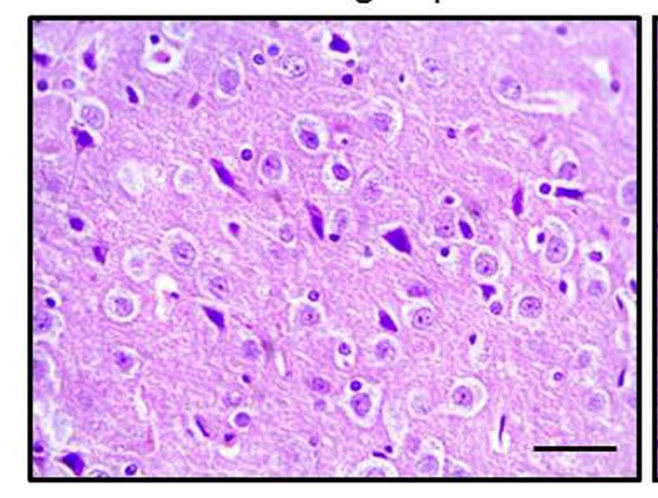

A

C

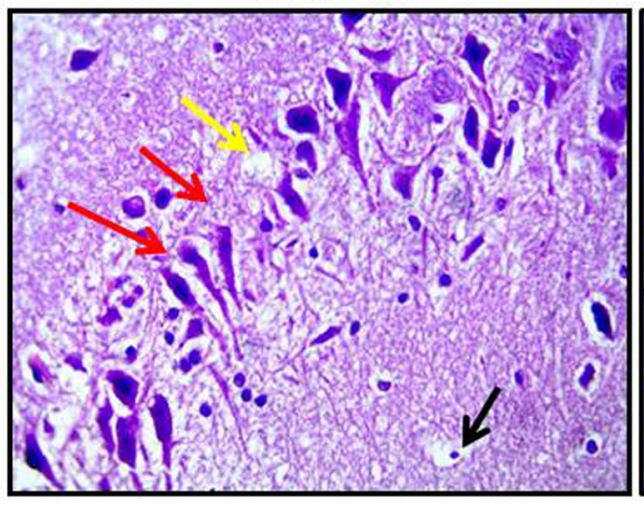

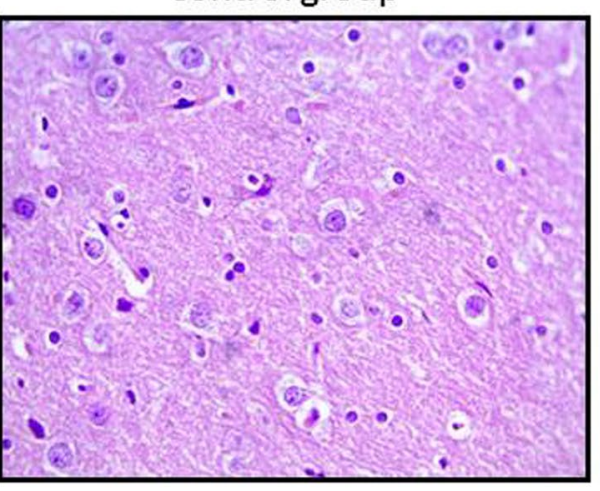

TQ treated SN
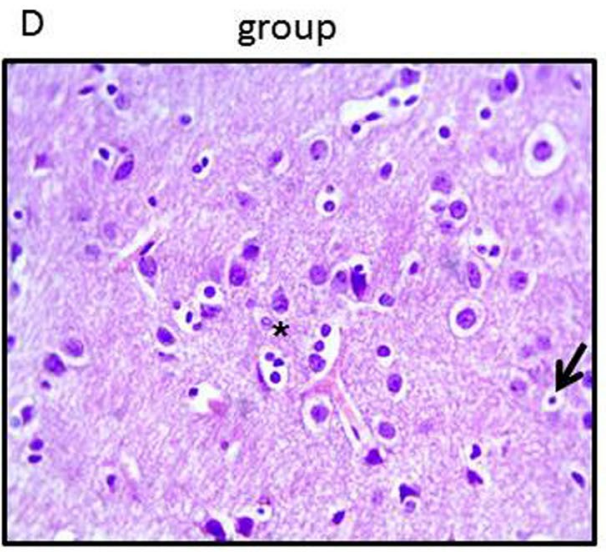

Figure 1. Microscopic images of brain tissues stained with hematoxylin/eosin from control (A) TQ treated control group (B) SN group (C) and TQ treated SN group. (D) Black arrows represented apoptosis. Yellow arrows represented vacuolation. Red arrows represented degradation and shrinkage of neuronal cells. Asterisks represented satellitosis. Images were taken under X400 magnification and scale bar represented $50 \mu \mathrm{m}$.

to acquire morbidity effects such as cancer especially brain cancer, leukemia, and nasopharyngeal tumors due to its oxidative properties ${ }^{5}$. The proposed sub-cellular mechanism for such cancer initiation property is that $\mathrm{SN}$ may form carcinogenic nitrosamines during charring or overcooking meat, under acidic conditions of the stomach or during the acidity produced in curing process of preserved meats ${ }^{6}$.

The human diet is an crucial component for safeguarding against the upgrowth of cancers. Some new trends in cancer chemotherapy recommended the use of some natural products ${ }^{7}$. One important natural product is Nigella sativa oil, frequently recognized as black cumin. This volatile oil is reported to include $30-48 \%$ thymoquinone (TQ), the main active ingredient ${ }^{8,9}$. TQ is important in relieving oxidative stress, improve the neuronal survival and therapeutic effects for the treatment of central nervous system tumors ${ }^{7,10,11}$. Oxidative stress was blamed for the harmful effects of higher concentrations of sodium nitrite ${ }^{12}$.

According to our best of knowledge, there is a lack in research for nutritional treatment of oxidative stress produced by the sodium nitrite. In addition, the molecular mechanism of the brain toxicity of sodium nitrite is rarely studied. Keeping in mind that, there is a great incidence of brain cancer among teens that may be linked to repeated intake of processed food with high amount of preservatives. Therefore, we investigated the molecular mechanism of chronic oral administration of SN on the brain tissues of rats and the protective role of thymoquinone. We concentrated on four major mechanisms; oxidative stress, inflammation, fibrosis and apoptosis.

\section{Results}

Effect of sodium nitrite (SN) and thymoquinone (TQ) on brain tissue structure. Microscopic pictures of brain tissue stained with hematoxylin/eosin from control groups show normal neurons and glial cells. Meanwhile, hippocampal sections from sodium nitrite group show excessive neuronal degeneration, shrinkage (red arrows) and vacuolation (yellow arrow). Exposure of sodium nitrite group to TQ ameliorated most of these effects (Fig. 1).

Effect of SN and TQ on the concentration levels of oxidative stress markers in brain tissue. Continuous exposure of rats to SN led to in marked increase (around 2.6 times, Supple Fig. 1A) in the brain concentrations of malondialdehyde (MDA). Besides, it lead to marked decreases (about 1.75 times, Supple Fig. 1B) in 

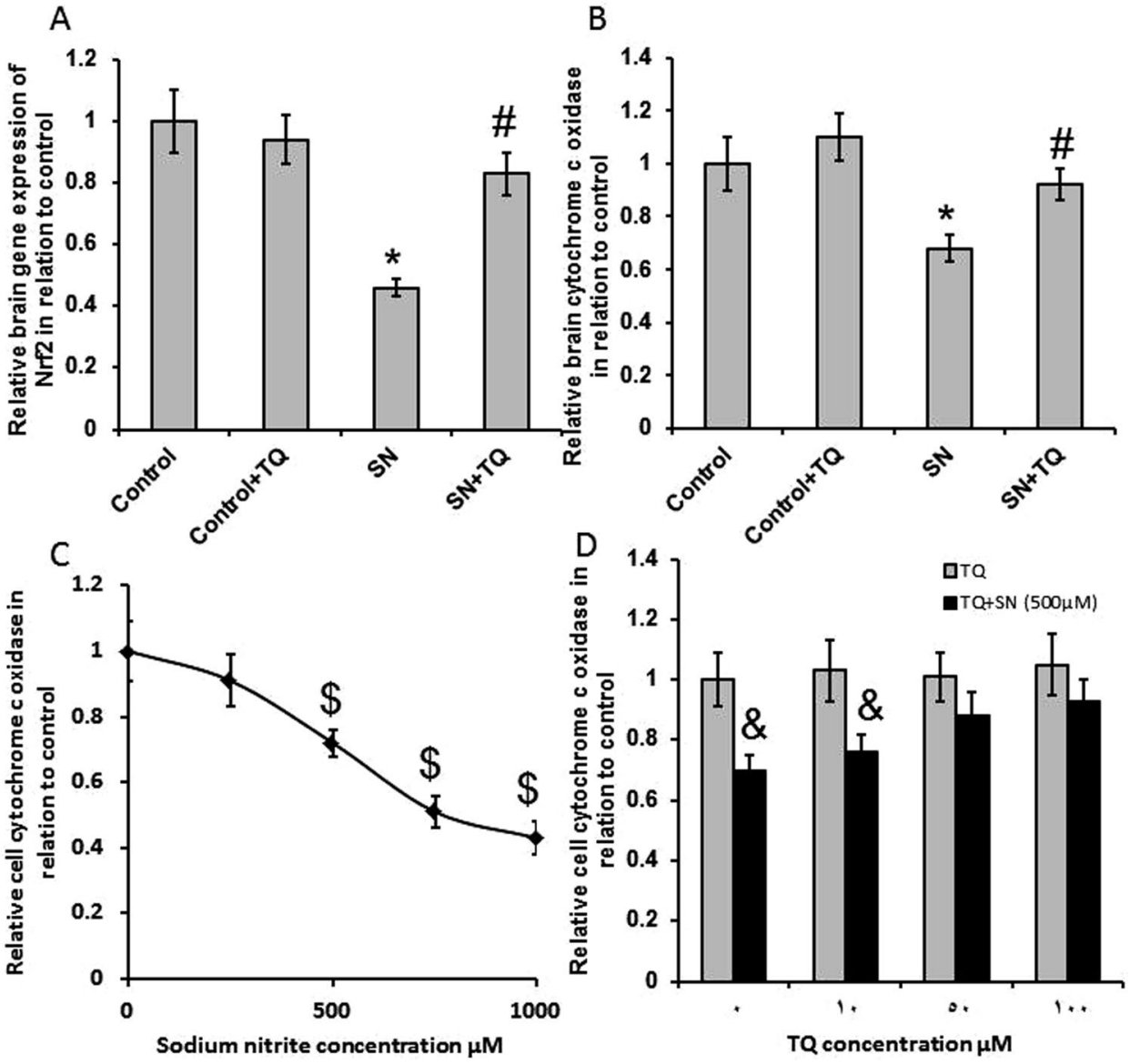

Figure 2. Effect of continuous oral exposure of SN; $80 \mathrm{mg} / \mathrm{kg} /$ day, only or concomitantly with TQ; $50 \mathrm{mg} / \mathrm{kg} / \mathrm{day}$, for 12 weeks on brain tissue gene expression of $\operatorname{Nrf2}(\mathbf{A})$ and brain tissue levels of cytochrome c oxidase. (B) Moreover, treatment of cultivated brain cells with SN resulted in the reduction of cytochrome c oxidase in a concentrationdependent manner. (C) However, concomitant administration of SN and TQ, inhibited reduction of cytochrome c oxidase (D) in a concentration-dependent manner. *Significant difference as compared with the control groups

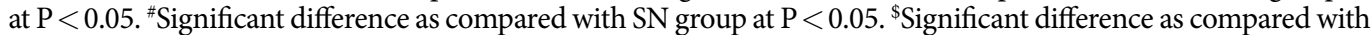
H19-7/IGF-IR cells treated with SN at $\mathrm{p}<0.05$. ${ }^{\circledR}$ Significant difference as compared with H19-7/IGF-IR cells treated with TQ alone at $\mathrm{p}<0.05$.

brain levels of reduced glutathione $(\mathrm{GSH})$ comparing to the control group $(\mathrm{p}<0.05)$. On the contrary, exposure of brain tissues of rats to TQ lead to significant reductions of MDA levels in a concentration-dependent manner (Supple Fig. 1C,D). Moreover, exposure of brain tissues of rats to TQ led to significant elevation of the activities of GSH in the sodium nitrite group. The control group of rats had no influence (Supple Fig. 1E,F).

Effect of $\mathrm{SN}$ and TQ on the concentration levels of $\mathrm{Nrf2}$ and markers concentration levels of mitochondrial oxidative stress in brain tissue. Continuous exposure of rats to SN led to significant decrease of the gene expression of Nrf2 and cytochrome c oxidase activity in brain tissues (around 1.75 times, Fig. $2 A, B)$ relative to the control group $(\mathrm{p}<0.05)$. Statistical analysis showing about $59 \%$ and $38 \%$ reduction in $\mathrm{Nrf2}$ gene expression and cytochrome c oxidase in brain tissue in rats received $\mathrm{SN}$ respectively relative to the control group. Exposure of brain cells of rats to $\mathrm{SN}$ led to significant, concentration-dependent decrease of cytochrome c oxidase concentrations in brain tissue. However, exposure of brain tissues to TQ lead to significant, concentration-dependent restoration of cytochrome c oxidase concentrations in brain tissue (Fig. 2).

Effect of chronic administration of SN and TQ on pro-inflammatory cytokines in brain tissue concentrations. Continuous exposure of rats to $\mathrm{SN}$ led to 2.6-fold elevation in the gene expression of NF- $\kappa B, 1.78$-fold elevation in concentration of Tumor necrosis factor type alpha; TNF- $\alpha$, in brain tissues of rats. In addition, we found 1.78-fold increase in the brain tissue concentrations of interleukin type 1beta; IL-1 $\beta$ and 1.48-fold decrease in the brain tissue concentrations of interleukin type 10; IL-10 (Fig. 3C), in rats exposed SN relative to the control rats. Exposure of rats to TQ blocked sodium nitrite-induced elevation in TNF- $\alpha$ and pro-inflammatory cytokines in brain tissue and reduction of IL-10, but it did not affect the control rats (Fig. 3D).

Effect of SN and TQ on pro-inflammatory cytokines in cultivated brain cells levels. Exposure of cultivated brain cells to SN led to elevation of the pro-inflammatory cytokines mediators; Tumor necrosis factor 
A

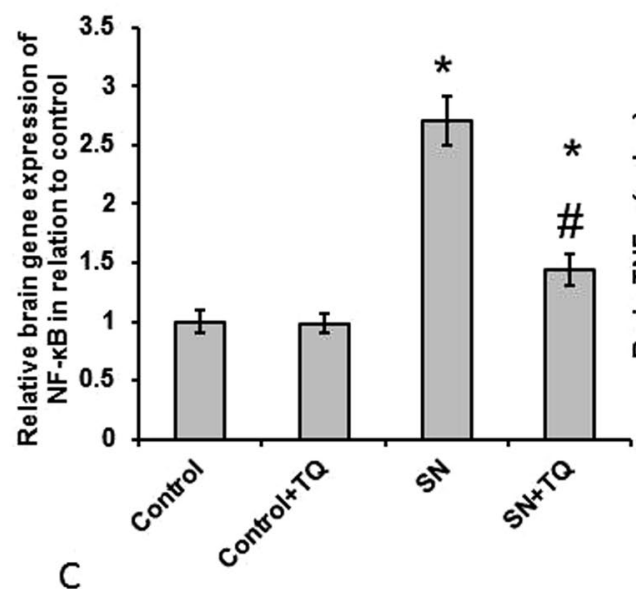

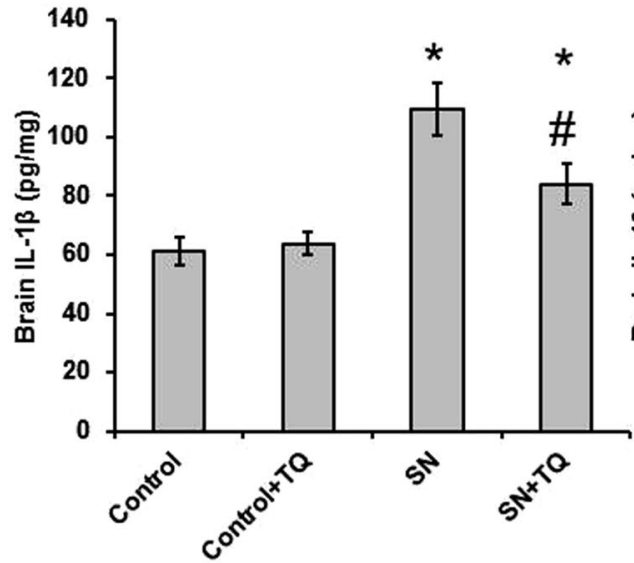

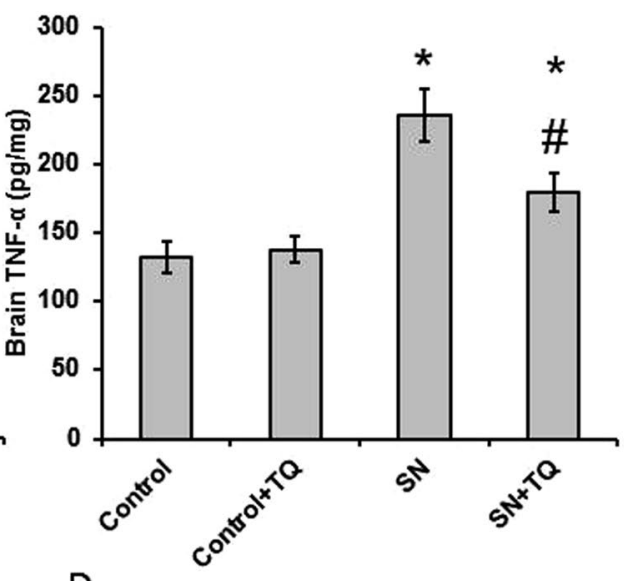

D

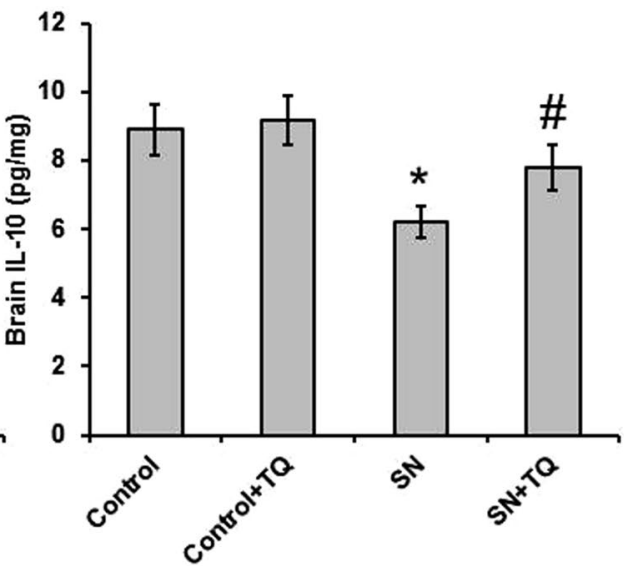

Figure 3. Effect of continuous oral exposure of $\mathrm{SN} ; 80 \mathrm{mg} / \mathrm{kg} /$ day, only or concomitantly with TQ; $50 \mathrm{mg} / \mathrm{kg} / \mathrm{day}$, for 12 weeks on brain gene expression of nuclear factor $N F-\kappa B(\mathbf{A})$ protein levels of tumor necrosis factor (TNF)- $\alpha$ (B) interleukin (IL) $1 \beta$ (C) and IL-10. (D) *Significant difference as compared with the control groups at $\mathrm{P}<0.05$. \#Significant difference as compared with $\mathrm{SN}$ group at $\mathrm{P}<0.05$.

type alpha; TNF- $\alpha$ (Fig. 4A) and interleukin type 1beta (IL1 $\beta$ ) (Fig. 4C) in a concentration-dependent manner and reduction of the concentration of interleukin type 10 (IL-10) (Fig. 4E) in a concentration-dependent manner. However, exposure of brain cells to $500 \mu \mathrm{g}$ of $\mathrm{SN}$ in combination with TQ, blocked increased concentrations of TNF- $\alpha$ (Fig. 4B) and IL1 $\beta$ (Fig. 4D) and reduced concentration of IL-10 in a concentration-dependent manner (Fig. 4F).

Effect of continuous administration of SN and TQ on acute inflammation phase in brain tissue levels. Continuous exposure of rats to SN led to 2-fold elevation of the concentration of c-reactive protein; CRP, in brain tissues of rats. However, the activity of CRP in rats exposed to both SN and TQ still significantly greater than that in the control rats. Addition of $500 \mu \mathrm{g}$ of SN to cultivated brain cells caused elevation of the concentration of the CRP in a concentration-dependent manner. However, treating brain cells with $500 \mu \mathrm{g}$ of SN in combination with TQ, inhibited elevated levels of CRP in a concentration-dependent manner (Supple Fig. 2).

Effect of continuous exposure to SN and TQ on fibrosis factors in brain cells concentrations. Continuous oral exposure of rats to $\mathrm{SN}$ led to 2.46-fold increase in concentration of transforming growth factor beta1; TGF- $\beta 1$, in the brain tissues of rats (Fig. $5 \mathrm{~A}$ ). However, the activity of TGF- $\beta 1$ in rats exposed to both SN and TQ is obviously greater than the control group. Exposure of cultivated brain cells to SN led to increase of the levels of TGF- $\beta 1$ in a concentration-dependent manner (Fig. 5B). However, concomitant addition of $500 \mu \mathrm{g}$ of SN and TQ, inhibited elevated concentrations of TGF- $\beta 1$ (Fig. $5 \mathrm{C}$ ) in a concentration-dependent manner.

Effect of continuous exposure to SN and TQ on apoptosis markers concentrations in brain cells. Continuous oral exposure to $80 \mathrm{mg} / \mathrm{kg} / \mathrm{day}$ of $\mathrm{SN}$ for twelve weeks led to 2.3 - and 1.74 -fold raising of brain tissue gene expression and enzyme activity of caspase-3; as an apoptosis marker, relative to the control rats. TQ significantly reduced sodium nitrite-induced elevation in the brain tissue activity of caspase-3. In addition, histological analysis of brain sections stained with anti-caspase-3 in CA3 pyramidal cell layer of hippocampal 

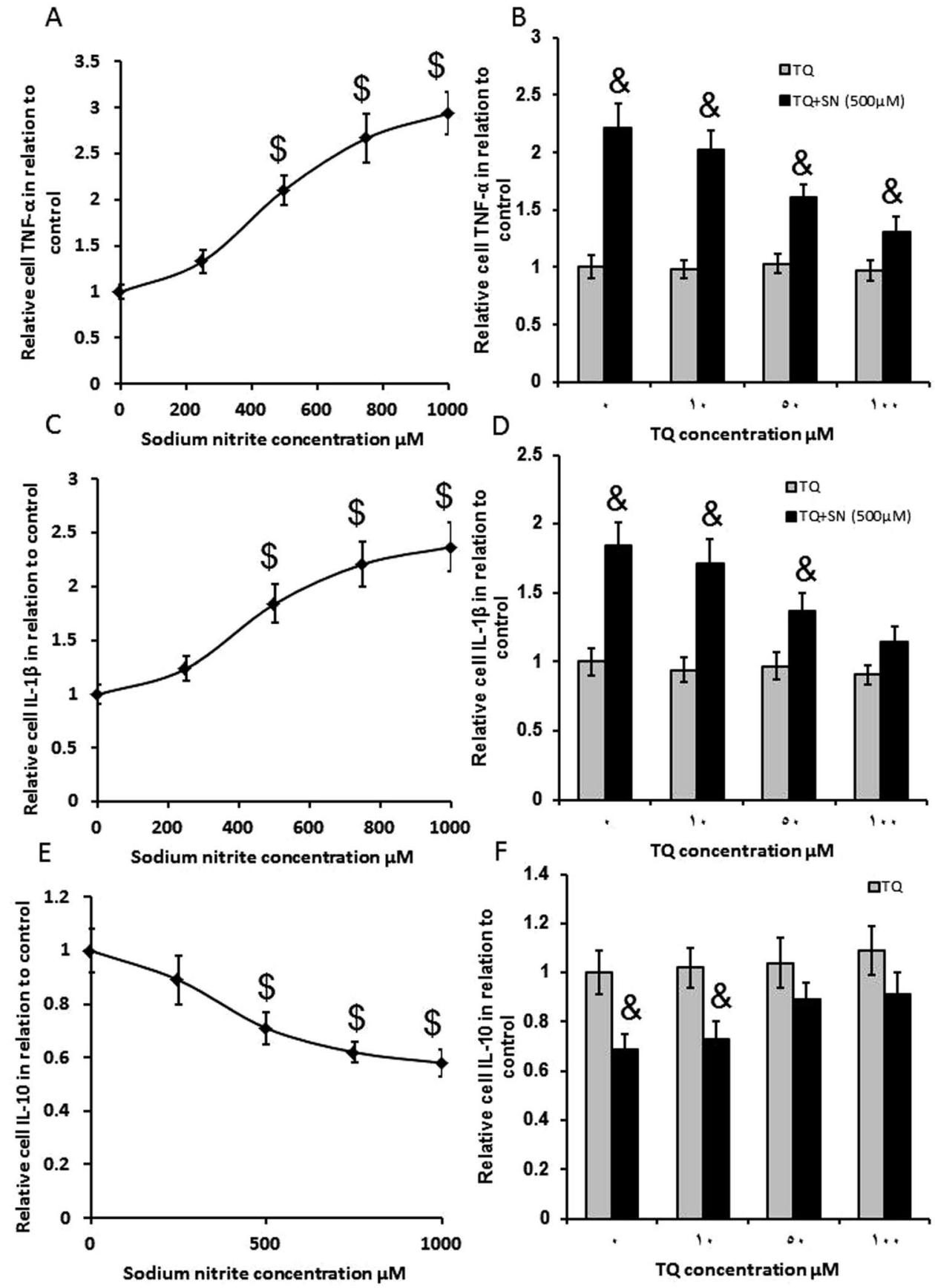

Figure 4. Effect of SN and TQ on pro-inflammatory cytokines in H19-7/IGF-IR brain cells. Exposure of cultivated brain cells to $\mathrm{SN}$ resulted in elevation of the pro-inflammatory cytokines mediators; Tumor necrosis factor type alpha; TNF- $\alpha(\mathbf{A}, \mathbf{B})$ and interleukin type 1 beta $(\operatorname{IL1} \beta)(\mathbf{C}, \mathbf{D})$ in a concentration-dependent manner and reduction of the concentration of interleukin type 10 (IL-10) (E,F) in a concentration- dependent manner. However, Exposure of brain cells to $500 \mu \mathrm{g}$ of SN in combination with TQ, inhibited changed concentrations of in a concentration-dependent manner. ${ }^{\$}$ Significant difference as compared with H19-7/IGF-IR cells treated with

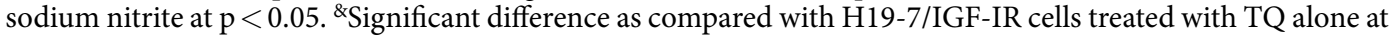
$\mathrm{p}<0.05$.

sections from control groups shows negative immunolabeling when compared with strong positive immunolabeling in SN group. Exposure of SN group to TQ attenuated caspase-3 immunolabeling (Fig. 6A-F).

However, the activity of caspase-3 in rats treated with both SN and TQ still significantly higher than the control group. Exposure of cultivated brain cells to SN led to increase of the caspase-3 in a concentration-dependent manner. However, concomitant addition of $500 \mu \mathrm{g}$ of SN and TQ, inhibited elevated concentrations of caspase-3 in a concentration-dependent manner. Cellular exposure to sodium nitrite resulted in the reduction of the cellular 


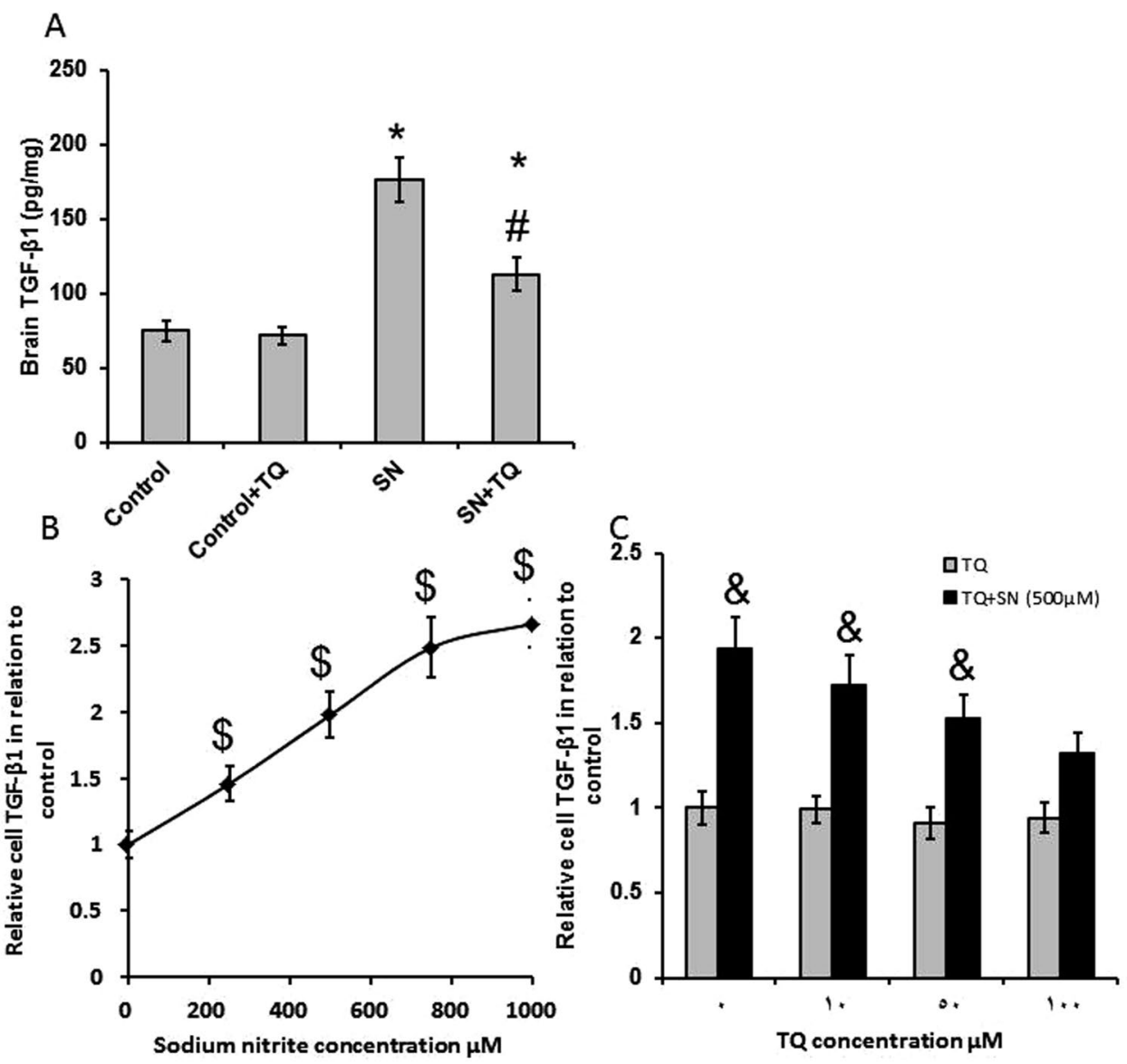

Figure 5. Effect of continuous oral exposure of $\mathrm{SN}$ and TQ on fibrosis factors in brain cells levels. Continuous exposure of rats with SN resulted in 2.46-fold increase in concentration of transforming growth factor beta1; TGF- $\beta 1$, in the brain tissues of rats. (A) Treatment of cultivated brain cells with SN resulted in the increase of the TGF- $\beta 1$ in a concentration-dependent manner. (B) However, concomitant administration of SN and TQ, inhibited elevated concentrations of TGF- $\beta 1$ (C) in a concentration-dependent manner. *Significant difference as compared with the control groups at $\mathrm{P}<0.05$. " Significant difference as compared with SN group at $\mathrm{P}<0.05$. ${ }^{\$}$ Significant difference as compared with H19-7/IGF-IR cells treated with SN at $\mathrm{p}<0.05$. ${ }^{\text {"Significant difference }}$ as compared with H19-7/IGF-IR cells treated with TQ alone at $\mathrm{p}<0.05$.

survival in a concentration-dependent manner. However, concomitant addition of $500 \mu \mathrm{g}$ of $\mathrm{SN}$ and TQ increases the survival rate of cultivated brain cells (Fig. 7A-D).

\section{Discussion}

The principle conclusion is that thymoquinone (TQ) attenuated sodium nitrite (SN) brain tissues damage in rats feed with SN. Our results explained that conclusion via multiple mechanisms including; First, TQ reduces oxidative stress induced by SN, as indicated by decreasing the level of MDA. Second, TQ blocks the inhibition induced by SN for the antioxidant activity as set out by retrieving the level of reduced glutathione. Third, TQ blocks increased level of pro-inflammatory cytokines such as TNF- $\alpha$ and IL1 $\beta$ induced by SN in brain tissues. Forth, TQ inhibits deactivated function of mitochondria induced by SN as indicated by retrieving cytochrome c oxidase activity. Finally, TQ decreases increased apoptosis marker levels induced by SN, caspase-3 in the brain tissues. According to the best of our many readings, it is the first time to study the cerebral-protection role of TQ for brain cancer induced by $\mathrm{SN}$ in rats. It has been previously published that molecular mechanism of the action of thymoquinone to remediate the pro-inflammatory mediator where TQ activates the Nrf2/ARE antioxidant mechanisms in its anti-inflammatory activity ${ }^{13}$. TQ inhibits lipopolysaccharide (LPS)-induced neuroinflammation through interference with NF- $\kappa B$ signaling ${ }^{13}$.

The molecular mechanism of the brain toxicity of SN is rarely studied. There are some attempts to show the histological changes of the brain tissues after chronic ingestion of $\mathrm{SN}^{14,15}$ and neuronal toxicity of nitrogen oxide in the brain tissue ${ }^{16,17}$. There is a great incidence of brain cancer between teens that may be linked to the increased ingestion of preserved and processed meats. According to the American Brain tumor Association, in December 2015 , brain cancer is the first most cancer among teens. Around 70,000 people, aged between 15-39, are identified with cancer every year in USA-about $5 \%$ of cancer cases are USA. The widely spread cancers for those between $15-39$ years old are brain and CNS cancers ${ }^{18-20}$. The 5-year rate of survival for brain cancer is about $33 \%$ in USA. 

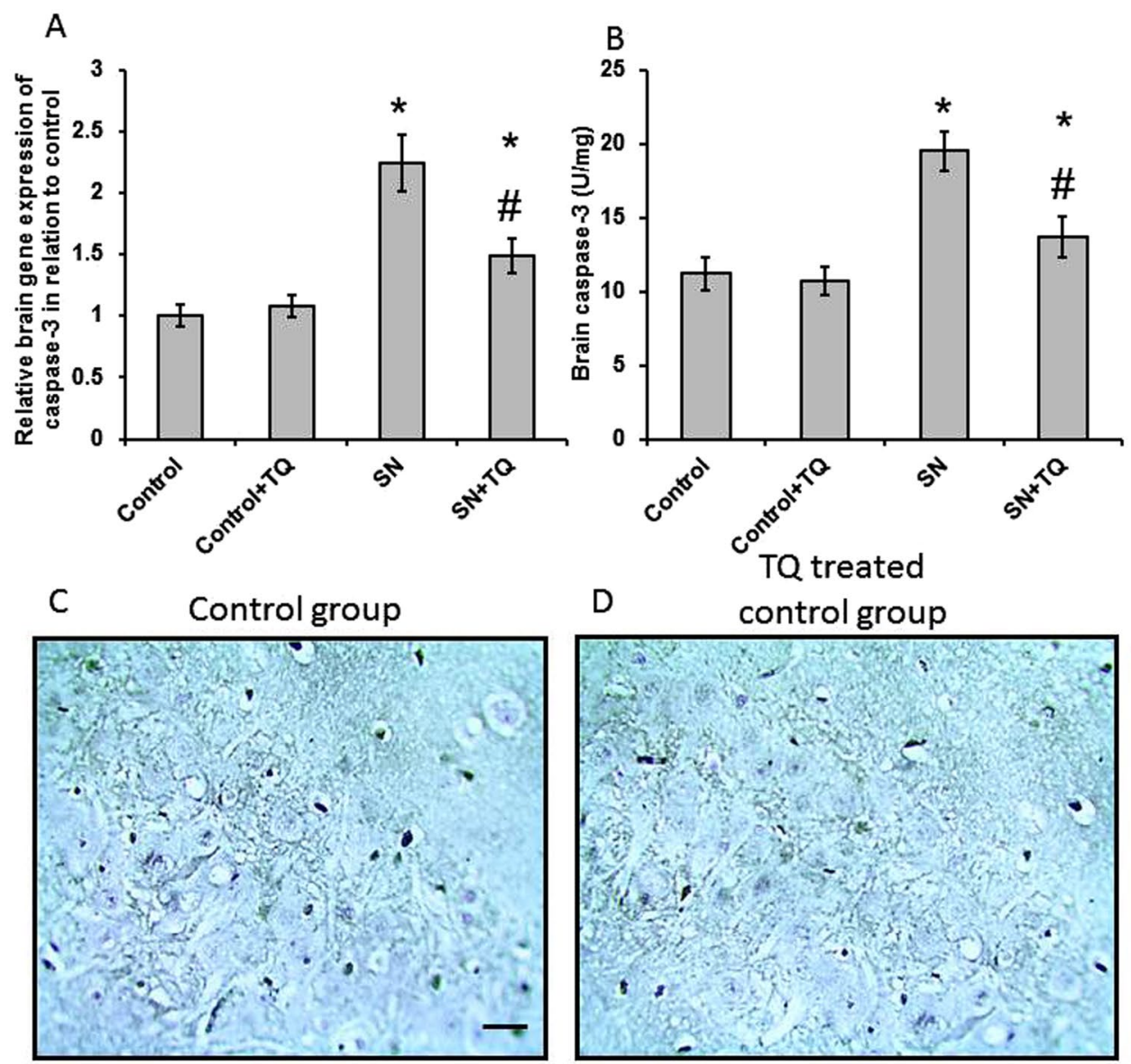

$\mathrm{D}$

TQ treated

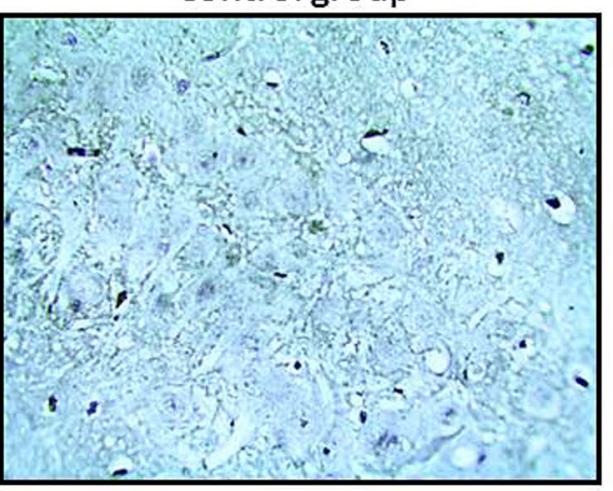

$\mathrm{E}$

SN group

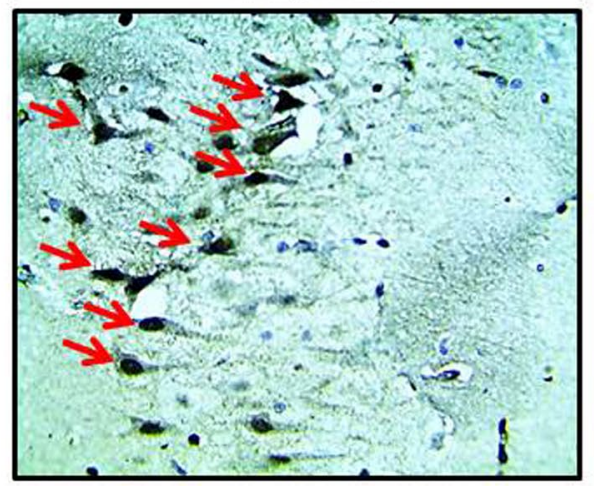

F

\section{TQ treated SN}

group

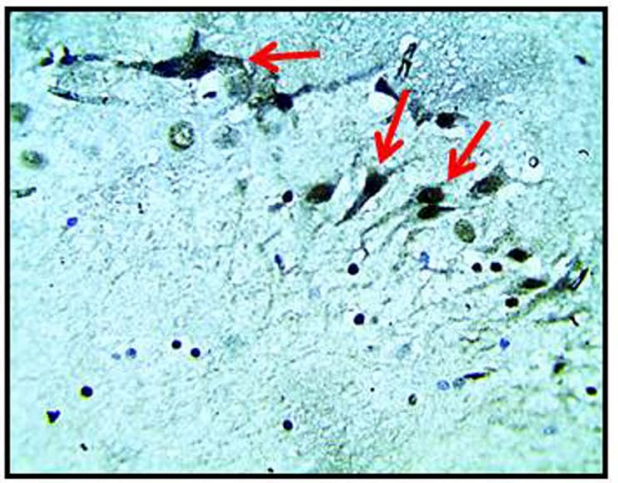

Figure 6. Effect of oral $80 \mathrm{mg} / \mathrm{kg}$ of SN only or concomitantly with $50 \mathrm{mg} / \mathrm{kg}$ TQ for 12 weeks on brain tissue gene expression (A) and enzyme activity (B) of caspase-3 (A) in vivo. Microscopic images of brain tissues immunostained with anti-caspase-3 antibodies from control (C) TQ treated control group (D) SN group (E) and TQ treated SN group. (F) Red arrows represented strong positive immunolabeling with caspase-3. Images were taken under X400 magnification and scale bar represented $50 \mu \mathrm{m}$. *Significant difference as compared with the control groups at $\mathrm{P}<0.05$. "Significant difference as compared with $\mathrm{SN}$ group at $\mathrm{P}<0.05$.

We studied the subcellular mode of action of continuous oral exposure to SN on the tissues of the brain in rats. Moreover, we explored the defensive effect of TQ against oxidative stress and pro-inflammatory mediators. SN is an important and a commonly used food preservative. Nitrite salts effectively retards the production of neurotoxic protein produced by the bacterium Clostridium botulinum in meat, effectively develops both the typical flavor and color of cured meat, slows up the oxidative rancidity upon storage, prevents the production of the characteristic bad flavor upon over warming, and maintains the flavors of both spice and smoke during cooking ${ }^{21,22}$. Despite the recent tremendous effort to decrease or even control dietary nitrite ingestion due to the proved carcinogenic $\mathrm{N}$-nitrosamines produced compounds inside the body, till now no general agreement 

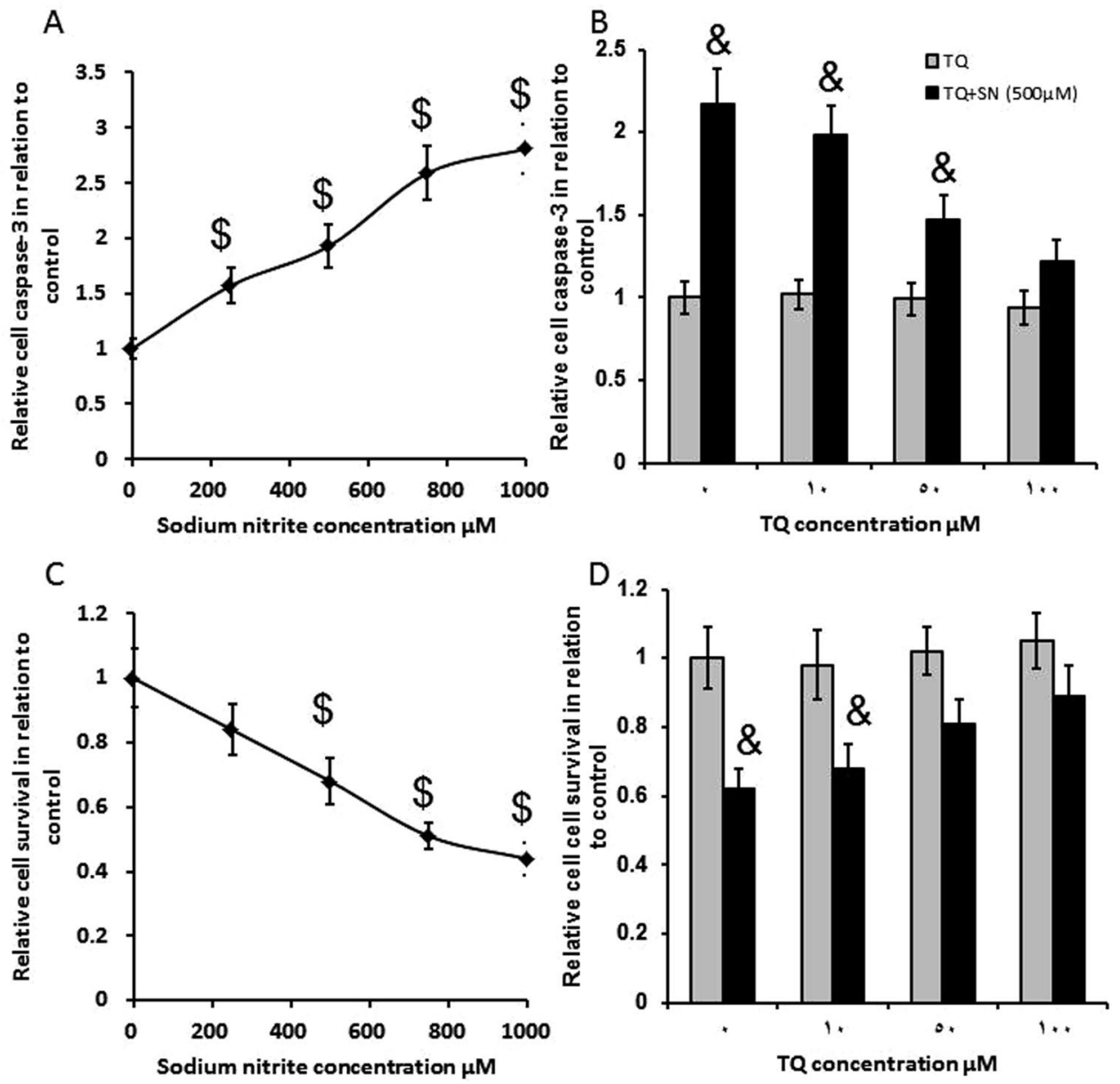

Figure 7. Exposure of cultivated brain cells to SN resulted in the increase of the caspase- 3 in a concentrationdependent manner. (A) However, concomitant administration of $500 \mu \mathrm{g}$ of $\mathrm{SN}$ and TQ, inhibited elevated concentrations of caspase-3 (B) in a concentration-dependent manner. Exposure SN led to reduction of the survival rate of cultivated brain tissue in a concentration-dependent manner. (C) However, concomitant exposure of SN and TQ inhibited reduction of the cellular survival rate (D). \$Significant difference as compared with H19-7/IGF-IR cells treated with SN at $\mathrm{p}<0.05$. ${ }^{\circledR}$ Significant difference as compared with H19-7/IGF-IR cells treated with TQ alone at $\mathrm{p}<0.05$.

has been adopted to point out that nutritional origin of nitrite is not safe. Since 1983, many researches linking between $\mathrm{N}$-nitrosamines and many types of cancers ${ }^{23}$. The passive significance of nitrite compounds urge the official authorities to control its use and limit its concentrations in drinking water and food, especially in processed meats. Meanwhile, some reports showed the beneficial effect of the nitrite salts in preventing the kidney injury and brain ischemia ${ }^{24,25}$.

In the current study, we found significant decreases in both reduced glutathione levels and cytochrome c oxidase activity in brain tissues chronically exposed to SN. Moreover, we discover markedly increase in malondialdehyde stress marker present in brain tissues of these rats. In parallel, in other research, SN showed hippocampal and cerebellar histological modifications leading to behavioral reflections induced by brain ischemic reduced oxygen and reduction of the brain weights in rats ${ }^{15,26}$. These findings showed brain impairment consequences for SN on brain tissues in rats, which are coordinated with a former finding that showed the number of neurons with lesions was considerably higher in animals treated with nitrite salts in areas of the hippocampus CA2, CA3, and $\mathrm{CA} 4^{15}$.

There is an intimate connection between food and disease that has been noticed throughout the history. Treatment and/or prophylaxis through sanitary production of nutrients and feeding traditions has been explored in spiritual and urban articles for a very long time. Therefore, we pursue for exploring the role of TQ, the principle active component of Nigella sativa oil, in rat model treated with $\mathrm{SN}$ for long time. Nigella sativa oil is claimed to have antagonistic effect against obesity, bacterial infection, increased blood sugar level, increased blood pressure, bronchial asthma, diarrhea and inflammation. Besides, it has a good effect for immune system and has neuro- and cardio-protective and antioxidant effects ${ }^{27}$. Administration of TQ for three months ameliorates both the elevated concentrations of creatinine and urea nitrogen in blood samples of rats treated with SN but had no effect on the control group ${ }^{8}$. Nigella sativa oil, extensively utilized as a dietary supplement, has been correlated with reduced 
concentrations of total cholesterol in blood, triglycerides, and/or elevated levels of high-density lipoprotein cholesterol (HDL-C) ${ }^{27}$. According to the best of our readings, no previous study has yet explored the powerful useful effects of Nigella sativa oil in blocking brain impairment induced by SN. We pursued exploring the subcellular mechanism of neuroprotective role of Nigella sativa oil.

In vivo, the produced reactive nitrogenous compounds due to the ingestion of nitrite salts is regarded as the main cause for many tissues damage. Nitrite salts triggers oxidative degeneration of lipids, DNA injuries and enzyme deactivation. MDA, high oxidative stress marker, attributes to the oxidative cellular toxicity of nitrite ${ }^{28}$. It has been previously explored that $\mathrm{SN}$ can make chemical interaction with proteinaceous ingredients in the stomach producing carcinogenic nitrosamines and reactive radicals, which may elevate lipid peroxidation tendency, leading to deleterious effects in different organs including liver ${ }^{12}$. Moreover, the consequence of consuming the nitrite salt for a long time on the energy production mitochondrial activity, specified by cytochrome c oxidase, was explored in our research. Mitochondrial cytochrome c oxidase enzyme is the last stage in the electron transfer in the electron transport chain inside mitochondria. It is fundamental for the production of aerobic ATP through stimulation of electron transfer using cytochrome $c$, a mitochondrial metalloenzyme, to oxygen in molecular state using copper element ${ }^{29}$. We found a marked reduction in the mitochondrial cytochrome $c$ oxidase enzymatic activity in rats that chronically exposed to SN. Previously, many researches proved that the main reasons for the down-regulation in the enzymatic activity of cytochrome coxidase is related to both reactive oxygen species and apoptosis as well ${ }^{30-32}$.

Our results elucidated marked increase in oxidative stress that was occurred along with marked decrease in levels of cytochrome c oxidase in rats treated with SN. Meanwhile, we discovered the importance of using Nigella sativa oil in retrieving brain cytochrome c oxidase activity in rats. Hence, Nigella sativa oil was expected to be beneficial in decreasing the oxidative stress, deactivation of mitochondria and DNA fragmentation caused by SN.

\section{Methods}

All methods are approved by the ethical research project committee in University of Tabuk (UT-46-10-2018) and performed in accordance with its relevant guidelines and regulations.

Animals and their exposures outlines. The methods are approved by the ethical research project committee in University of Tabuk (UT-46-10-2018) and performed in accordance with its relevant guidelines and regulations.

We used forty healthy adult (3-4 months) male Sprague Dawley (SD) rats. The average weight was 120$140 \mathrm{~g}$. Rats were kept in polycarbonate cages padded with paper in individual manner and covered by stainless steel wire. The ethical committee in Faculty of Pharmacy, University of Mansoura, Mansoura, Egypt (2012-32), approves all animal facilities and animal protocols. Water and food are given in a free access manner. Rats were kept in animal facilities under controlled $12 / 12 \mathrm{~h}$ light/dark cycle with controlled temperature and humidity. All exposures were given using oral gavage. The forty rats were randomly distributed into four groups (ten rats each):

(A) Control group: Rats received normal saline daily for twelve weeks.

(B) Treated control group: Rats received $50 \mathrm{mg} / \mathrm{kg}$ of TQ daily for twelve weeks.

(C) SN group: Rats received daily $80 \mathrm{mg} / \mathrm{kg} \mathrm{SN}$ orally for twelve weeks.

(D) $\mathrm{SN}+\mathrm{TQ}$ group: Rats received $50 \mathrm{mg} / \mathrm{kg}$ of TQ followed by $80 \mathrm{mg} / \mathrm{kg}$ of SN daily for twelve weeks.

The concentration and time course used for SN and TQ were in the range of those used in other studies ${ }^{8}$.

Animal scarification and collection of samples. The method is approved by the ethical research project committee in University of Tabuk (UT-46-10-2018) and performed in accordance with its relevant guidelines and regulations.

As previously described ${ }^{33}$, exposure to both TQ and SN was interrupted $24 \mathrm{~h}$ before sacrificing by cervical dislocation in the morning after overnight fasting. Brains were treated the same as previously described ${ }^{33}$ till we get the homogenized tissue and stored at $-80^{\circ} \mathrm{C}$ until using. Blood from the trunk was collected and were treated the same as previously described ${ }^{33}$ till we get the homogenized tissue and stored at $-80^{\circ} \mathrm{C}$ until using.

Morphologic analysis and immunohistochemistry. The method is approved by the ethical research project committee in University of Tabuk (UT-46-10-2018) and performed in accordance with its relevant guidelines and regulations.

Brain tissues were cut into five-micrometer sections and stained with hematoxylin/eosin. For immunohistochemical analyses, sections were incubated with monoclonal anti-caspase-3 (Sigma Aldrich Chemicals Co., St Louise, MO, US) at $4^{\circ} \mathrm{C}$. Slides were counterstained with hematoxylin.

Cell culture. The methods are approved by the ethical research project committee in University of Tabuk (UT-46-10-2018) and performed in accordance with its relevant guidelines and regulations.

H19-7/IGF-IR rat brain/hippocampus cells (ATCC ${ }^{\circledR}$ No. CRL-2526 ${ }^{\mathrm{TM}}$ ) were cultivated in a T25 flask lined with collagen for $1 \mathrm{hr}$ at $24^{\circ} \mathrm{C}$. The cells were passaged two times per week. The cells between passages three and eight were used.

Assessment of oxidative stress. The methods are approved by the ethical research project committee in University of Tabuk (UT-46-10-2018) and performed in accordance with its relevant guidelines and regulations.

Oxidative stress was estimated using the following parameters: 


\begin{tabular}{|l|l|l|}
\hline Gene & Forward Primer & Reverse Primer \\
\hline$N r f 2$ & $5^{\prime}$-GAGACGGCCATGACTGAT-3' & 5'-GTGAGGGGATCGATGAGTAA-3' $^{\prime}$ \\
\hline$N F-\kappa B$ & $5^{\prime}$-ACCCTGACCTTGCCTATTTG-3' & 5'-GAAAAGCTGTAAACATGAGCCG-3' $^{\prime}$ \\
\hline caspase-3 & 5'-CGTCTGTGCTCCAGGCTTC-3' $^{\prime}$ & 5'-TGTGAGTTCCTTCCTTTCTTTGTG-3' $^{\prime}$ \\
\hline GAPDH & 5'-CCATCAACGACCCCTTCATT-3' $^{\prime}$ & 5'-CACGACATACTCAGCACCAGC-3' $^{\prime}$ \\
\hline
\end{tabular}

Table 1. Primers used for measuring the genetic expression of $N r f 2, N F-\kappa B$, caspase- 3 and GAPDH.

Brain level of malondialdehyde (MDA). The method is approved by the ethical research project committee in University of Tabuk (UT-46-10-2018) and performed in accordance with its relevant guidelines and regulations.

We used thiobarbituric acid to measure brain level of malondialdehyde (MDA) as described previously ${ }^{34}$. Briefly, we precipitated all proteins using trichloroacetic acid. Then, we added thiobarbituric acid to MDA in samples in buffer system to form the chromophore thiobarbituric acid-reactive substance, which is measured at $532 \mathrm{~nm}$.

Brain reduced glutathione (GSH). The method is approved by the ethical research project committee in University of Tabuk (UT-46-10-2018) and performed in accordance with its relevant guidelines and regulations.

As previously described ${ }^{35}$, brain reduced glutathione (GSH) levels were determined colorimetrically at $412 \mathrm{~nm}$.

ELISA determination. The method is approved by the ethical research project committee in University of Tabuk (UT-46-10-2018) and performed in accordance with its relevant guidelines and regulations.

As previously described ${ }^{36}$, Brain levels of Tumor necrosis factor alpha, Interleukin-1 beta, Interleukin 10, Tumor growth factor beta- 1 and c-reactive protein were assessed using ELISA kits according to the manufacturers' instructions.

Determination of brain mitochondrial function. The method is approved by the ethical research project committee in University of Tabuk (UT-46-10-2018) and performed in accordance with its relevant guidelines and regulations.

As previously described ${ }^{34}$, cytochrome c oxidase activity was assessed using available kit (Sigma-Aldrich).

Determination of caspases activity. The method is approved by the ethical research project committee in University of Tabuk (UT-46-10-2018) and performed in accordance with its relevant guidelines and regulations.

The enzymatic activity of caspase- 3 was assessed colorimetrically using commercially available kits by Spectronics Spectrophotometer.

Real-time PCR. The method is approved by the ethical research project committee in University of Tabuk (UT-46-10-2018) and performed in accordance with its relevant guidelines and regulations.

Gene expression of $N r f 2, N F-\kappa B$ and caspase-3 were measured as described previously by our group ${ }^{32}$. The used primers are mentioned in Table (1).

Statistical analysis. Values were calculated as mean values $+/-$ standard error. Normality was examined with the Kolmogorov-Smirnov test. One-way analysis of variance (ANOVA) was applied to compare the means between groups. Once differences between the means were found, and post-hoc Bonferroni correction tests were measured. Statistical computations were performed using SPSS version 20 (Chicago, IL, USA). Statistical significance was predefined as $\mathrm{P} \leq 0.05$.

\section{References}

1. Milkowski, A., Garg, H. K., Coughlin, J. R. \& Bryan, N. S. Nutritional epidemiology in the context of nitric oxide biology: a riskbenefit evaluation for dietary nitrite and nitrate. Nitric Oxide. 22(2), 110-119 (2010).

2. Sullivan, G. A. et al. Inhibition of Listeria monocytogenes using natural antimicrobials in no-nitrate-or-nitrite-added ham. J. Food Prot. 75(6), 1071-1076 (2012).

3. Sindelar, J. J. \& Milkowski, A. L. Human safety controversies surrounding nitrate and nitrite in the diet. Nitric Oxide. 26(4), 259-266 (2012).

4. Richard, A. M., Diaz, J. H. \& Kaye, A. D. Reexamining the risks of drinking-water nitrates on public health. Ochsner. J. 14(3), 392-398 (2014)

5. McKean-Cowdin, R. et al. Maternal prenatal exposure to nitrosatable drugs and childhood brain tumours. Int. J. Epidemiol. 32(2), 211-217 (2003).

6. Jakszyn, P. \& Gonzalez, C. A. Nitrosamine and related food intake and gastric and oesophageal cancer risk: a systematic review of the epidemiological evidence. World J. Gastroenterol. 12(27), 4296-4303 (2006).

7. Shahid, F., Farooqui, Z., Khan, A. A. \& Khan, F. Oral Nigella sativa oil and thymoquinone administration ameliorates the effect of long-term cisplatin treatment on the enzymes of carbohydrate metabolism, brush border membrane, and antioxidant defense in rat intestine. Naunyn Schmiedebergs Arch. Pharmacol. 391(2), 145-157 (2018).

8. Elsherbiny, N. M. \& El-Sherbiny, M. Thymoquinone attenuates Doxorubicin-induced nephrotoxicity in rats: Role of Nrf2 and NOX4. Chem. Biol. Interact. 223, 102-108 (2014).

9. Daryabeygi-Khotbehsara, R., Golzarand, M., Ghaffari, M. P. \& Djafarian, K. Nigella sativa improves glucose homeostasis and serum lipids in type 2 diabetes: A systematic review and meta-analysis. Complement. Ther. Med. 35, 6-13 (2017).

10. Beker, M., Dall, T. \& Elibol, B. Thymoquinone Can Improve Neuronal Survival and Promote Neurogenesis in Rat Hippocampal Neurons. Mol. Nutr. Food Res. 62(5), https://doi.org/10.1002/mnfr.201700768 (2018).

11. Farkhondeh, T., Samarghandian, S., Hozeifi, S. \& Azimi-Nezhad, M. Therapeutic effects of thymoquinone for the treatment of central nervous system tumors: A review. Biomed. Pharmacother. 96, 1440-1444 (2017). 
12. Hassan, H. A., Hafez, H. S. \& Zeghebar, F. E. Garlic oil as a modulating agent for oxidative stress and neurotoxicity induced by sodium nitrite in male albino rats. Food Chem Toxicol. 48(7), 1980-1985 (2010).

13. Petrova, E. B. et al. Effect of acute hypoxic shock on the rat brain morphology and tripeptidyl peptidase I activity. Acta Histochem. 118(5), 496-504 (2016).

14. Velagapudi, R. et al. Inhibition of neuroinflammation by thymoquinone requires activation of Nrf2/ARE signaling. int. Immunopharmacol. 48, 17-29 (2017).

15. Ribeiro, M. C. et al. Hippocampal and cerebellar histological changes and their behavioural repercussions caused by brain ischaemic hypoxia experimentally induced by sodium nitrite. Behav. Brain Res. 332, 223-232 (2017).

16. Kuzenkov, V. S. \& Krushinskii, A. L. Effect of Sodium Nitrite and L-NNA on the Outcome of Experimental Ischemic Stroke. Bull. Exp. Biol. Med. 159(2), 217-220 (2015).

17. Singh, B. K. et al. Neuronal Nitric Oxide Synthase Negatively Regulates Zinc-Induced Nigrostriatal Dopaminergic Neurodegeneration. Mol. Neurobiol. 54(4), 2685-2696 (2017).

18. Ostrom, Q. T. et al. American Brain Tumor Association Adolescent and Young Adult Primary Brain and Central Nervous System Tumors Diagnosed in the United States in 2008-2012. Neuro. Oncol. 18(Suppl 1), i1-i50, https://doi.org/10.1093/neuonc/nov297 (2016).

19. Zetterling, M., Berhane, L., Alafuzoff, I., Jakola, A. S. \& Smits, A. Prognostic markers for survival in patients with oligodendroglial tumors; a single-institution review of 214 cases. PLoS One. 12(11), e0188419 (2017).

20. Zapotocky, M., Ramaswamy, V., Lassaletta, A. \& Bouffet, E. Adolescents and young adults with brain tumors in the context of molecular advances in neuro-oncology. Pediatr. Blood Cancer. 65(2), https://doi.org/10.1002/pbc.26861 (2018).

21. Stokes, K. Y. et al. Dietary nitrite prevents hypercholesterolemic microvascular inflammation and reverses endothelial dysfunction. Am. J. Physiol. Heart Circ. Physiol. 296(5), H1281-8 (2009).

22. de La Pomélie, D., Santé-Lhoutellier, V., Sayd, T. \& Gatellier, P. Oxidation and nitrosation of meat proteins under gastro-intestinal conditions: Consequences in terms of nutritional and health values of meat. Food Chem. 243, 295-304 (2018).

23. Craddock, V. M. Nitrosamines and human cancer: proof of an association? Nature. 306(5944), 638 (1983).

24. Jung, K. H. et al. Early intravenous infusion of sodium nitrite protects brain against in vivo ischemia-reperfusion injury. Stroke. 37(11), 2744-2750 (2006).

25. Kelpke, S. S. et al. Sodium nitrite protects against kidney injury induced by brain death and improves post-transplant function. Kidney Int. 82(3), 304-313 (2012).

26. Ali, E. H. A., Ahmed-Farid, O. A. \& Osman, A. A. E. Bone marrow-derived mesenchymal stem cells ameliorate sodium nitriteinduced hypoxic brain injury in a rat model. Neural Regen. Res. 12(12), 1990-1999 (2017).

27. Sahebkar, A., Beccuti, G., Simental-Mendía, L. E., Nobili, V. \& Bo, S. Nigella sativa (black seed) effects on plasma lipid concentrations in humans: A systematic review and meta-analysis of randomized placebo-controlled trials. Pharmacol. Res. 106, 37-50 (2016).

28. Patsoukis, N. \& Georgiou, C. D. Effect of glutathione biosynthesis-related modulators on the thiol redox state enzymes and on sclerotial differentiation of filamentous phytopathogenic fungi. Mycopathologia. 163(6), 335-347 (2007).

29. Horn, D. \& Barrientos, A. Mitochondrial copper metabolism and delivery to cytochrome c oxidase. IUBMB Life. 60(7), 421-429 (2008),

30. Papadopoulou, L. C., Theophilidis, G., Thomopoulos, G. N. \& Tsiftsoglou, A. S. Structural and functional impairment of mitochondria in adriamycin-induced cardiomyopathy in mice: suppression of cytochrome c oxidase II gene expression. Biochem. Pharmacol. 57(5), 481-489 (1999).

31. Villani, G. \& Attardi, G. In vivo control of respiration by cytochrome c oxidase in human cells. Free Radic. Biol. Med. 29(3-4), 202-210 (2000)

32. Garcia, L., Welchen, E. \& Gonzalez, D. H. Mitochondria and copper homeostasis in plants. Mitochondrion. 19(Pt B), 269-74 (2014).

33. Alyoussef, A. \& Al-Gayyar, M. M. Thymoquinone ameliorates testicular tissue inflammation induced by chronic administration of oral sodium nitrite. Andrologia. 48(5), 501-508 (2016).

34. Sherif, I. O. \& Al-Gayyar, M. M. Antioxidant, anti-inflammatory and hepatoprotective effects of silymarin on hepatic dysfunction induced by sodium nitrite. Eur Cytokine Netw. 24(3), 114-121 (2013).

35. Al-Gayyar, M. M., Al Youssef, A., Sherif, I. O., Shams, M. E. \& Abbas, A. Protective effects of arjunolic acid against cardiac toxicity induced by oral sodium nitrite: effects on cytokine balance and apoptosis. Life Sci. 111(1-2), 18-26 (2014).

36. Alyoussef, A. \& Al-Gayyar, M. M. H. Cytotoxic and partial hepatoprotective activity of sodium ascorbate against hepatocellular carcinoma through inhibition of sulfatase-2 in vivo and in vitro. Biomed. Pharmacother. 103, 362-372 (2018),

\section{Acknowledgements}

The authors would like to acknowledge financial support for this work from the deanship of scientific research (DSR), University of Tabuk, Tabuk, Saudi Arabia, under the Grant Number S/1438/0014.

\section{Author Contributions}

Ahmed M. Hamdan; in vitro experiments, Mohammed M. Al-Gayyar; Concept and design, Mohamed Shams; data analysis, Udai Salamh Alshaman; in vitro experiments, Kousalya Prabahar; in vivo experiments, Alaa Bagalagel; final approval of the revised version to be published, Reem Diri; Concept and design, Ahmad O. Noor; in vivo experiments; Diena Almasri; in vivo experiments.

\section{Additional Information}

Supplementary information accompanies this paper at https://doi.org/10.1038/s41598-019-43568-x.

Competing Interests: The authors declare no competing interest.

Publisher's note: Springer Nature remains neutral with regard to jurisdictional claims in published maps and institutional affiliations.

Open Access This article is licensed under a Creative Commons Attribution 4.0 International License, which permits use, sharing, adaptation, distribution and reproduction in any medium or format, as long as you give appropriate credit to the original author(s) and the source, provide a link to the Creative Commons license, and indicate if changes were made. The images or other third party material in this article are included in the article's Creative Commons license, unless indicated otherwise in a credit line to the material. If material is not included in the article's Creative Commons license and your intended use is not permitted by statutory regulation or exceeds the permitted use, you will need to obtain permission directly from the copyright holder. To view a copy of this license, visit http://creativecommons.org/licenses/by/4.0/.

(C) The Author(s) 2019 